\title{
Germanica
}

\section{La hantise de la question chez Thomas Bernhard}

Und die Frage läßt ihm keine Ruhe...

\section{Catharina Wulf}

\section{(2) OpenEdition}

Journals

Édition électronique

URL : http://journals.openedition.org/germanica/1328

DOI : 10.4000/germanica.1328

ISSN : 2107-0784

Éditeur

Université de Lille

\section{Édition imprimée}

Date de publication : 30 juin 1998

Pagination : 135-144

ISBN : $9770984263203-22$

ISSN : 0984-2632

\section{Référence électronique}

Catharina Wulf, «La hantise de la question chez Thomas Bernhard », Germanica [En ligne], 22 | 1998, mis en ligne le 31 janvier 2012, consulté le 06 octobre 2020. URL : http://journals.openedition.org/ germanica/1328; DOI : https://doi.org/10.4000/germanica.1328

Ce document a été généré automatiquement le 6 octobre 2020.

(c) Tous droits réservés 


\title{
La hantise de la question chez Thomas Bernhard
}

\author{
Und die Frage läßt ihm keine Ruhe...
}

\author{
Catharina Wulf
}

1 Prenez n'importe quel livre de Thomas Bernhard et vous serez aussitôt noyé dans une cascade verbale ininterrompue, pleine d'accusations, de malédictions, de rage. Beaucoup de critiques y ont vu l'expression d'un univers profondément maudit, dénué de sens et d'espoir. On peut facilement arriver à cette conclusion si l'on se contente de survoler les écrits de l'Autrichien. Ouvrant l'un de ses romans au hasard on trouve d'emblée la thématique de la mort, du suicide, de l'échec, de la maladie ou de l'isolement de l'individu, pour ne citer que certaines caractéristiques de son écriture. Il faut cependant aller au-delà de cette vision pessimiste. Car, dans le tourbillon véhément de mots proférés, on ressent vivement la présence d'un Moi impitoyable, d'un Moi qui refuse de s'enfermer dans le désespoir. Que ce Moi souffre d'une maladie inguérissable ou de la perte d'un être aimé, il est loin de sombrer dans un état permanent de détresse. Regardons de plus près les exemples suivants, tirés du récit autobiographique Die Kälte (Le Froid) : «Il faut que le malade prenne lui-même, son affection en main [...] » (F 25)․․ Et plus loin, Bernhard dit à propos du décès de son aïeul aimé, l'écrivain Johannes Freumbichler: «mon grand-père, le poète, était mort; maintenant moi, j'avais le droit d'écrire, maintenant moi, j'avais la possibilité d'écrire moi-même des poèmes [...] » (F 37). Les deux citations révèlent un Bernhard cherchant à la fois à combattre sa tuberculose et à explorer ses propres exigences esthétiques après la mort de son grand-père. Il s'agit alors d'une personnalité tenace, toujours prête à s'engager dans une lutte, quelle qu'en soit l'issue. D'où vient-elle, cette rigueur absolue ? Et qui est ce Moi qui se cache derrière le maëlstrom verbal ?

2 Pour essayer de répondre à ces questions, il importe de considérer les récits autobiographiques de Bernhard, notamment Ein Kind (L'Enfant), Der Atem (Le Souffle)et Die Kälte (Le Froid). Il est trop facile de réduire l'écriture d'un auteur à sa biographie. Or, chez Bernhard, cette dernière se comprend aussi et surtout comme fiction (d'où mon utilisation du terme " récits autobiographiques »). Dans ces trois récits, nous trouvons 
un Moi, (le narrateur /Bernhard), qui a vécu des événements tragiques, tels que la Deuxième Guerre mondiale, le décès inattendu de son grand-père maternel, «le seul être réellement aimé " (S 11), la longue maladie de sa mère, suivie de sa mort prématurée ${ }^{2}$. À cela s'ajoutent la tuberculose, maladie contre laquelle Bernhard a lutté jusqu'à la fin de sa vie et les ténèbres autour de son père, que l'écrivain n'a jamais connu.

3 Lorsqu'on s'arrête sur ces faits tragiques, on parvient difficilement à dégager une vision optimiste des récits autobiographiques de l'Autrichien. Peut-être serait-on tenté de croire que, chez Bernhard, tout aboutit à une noirceur interminable. Malgré les moments douloureux qui ont assombri sa jeunesse, il s'est toujours désespérément accroché à la vie. Ainsi explique-t-il son sens de la combativité et la rigueur absolue qui le poussent à vouloir surmonter tous les obstacles.

4 Dans le premier récit autobiographique Ein Kind, cette rigueur, bien qu'étant encore l'expression d'une rêverie propre à l'enfant, s'annonce dès le début. Le petit Thomas, âgé de huit ans, est entrain de faire ses premiers tours sur le vélo de son tuteur. Encore «trop petit pour être effectivement assis sur la selle» (E 12), il refuse de renoncer à cette nouvelle activités. Ayant pédalé autour de l'église et de la Grand-Place, il décide de sortir du village pour rendre visite à sa tante Fanny qui habite à trente-six kilomètres de Traunstein. L'enfant est d'abord emporté par le goût de l'aventure : « Il leur faudrait bien reconnaître que contre les plus grands obstacles, les plus grandes résistances, j'arrivais pourtant toujours à mes fins et que j'étais vainqueur » (E 13). Pourtant après un certain temps, l'enthousiasme du petit Thomas commence à s'estomper. À bout de souffle, il se demande s'il est encore en mesure de poursuivre cette promenade démesurée. Tout à coup, il doit abandonner son projet d'aller à Salzbourg. La chaîne du vélo se rompt, le garçon fait un "saut désastreux » et se retrouve dans un fossé. En faisant demi-tour, il se sent triste et coupable parce qu'il a utilisé le vélo de son tuteur sans lui avoir demandé la permission. Or, plus tard, l'enfant parvient à se réconcilier avec lui-même en racontant l'accident à un ami :

Je jouissais moi-même de mon récit [...] et je m'exaltais à mesure que les mots se succédaient, en donnant à l'ensemble [...] une série d'accentuations qui étaient soit des exagérations destinées à pimenter tout le récit, soit même des inventions supplémentaires, pour ne pas dire : des mensonges (E 35).

5 Cette scène montre le goût de l'enfant pour transformer son malheur en fiction. Plus précisément, l'acte créateur lui permet d'oublier l'angoisse qu'il avait ressentie en poussant le vélo tout au long de son pénible retour. Par ailleurs, l'invention d'un récit anticipe sur le principe artistique, celui de "l'art de l'exagération» dont jouit le personnage Murau dans Extinction, roman dans lequel l'exagération se comprend comme un rempart contre une réalité insupportable, celle de la mort.

6 Si l'enfant parvient à embellir son malheureux accident de bicyclette, cet événement reste secondaire par rapport aux décès du grand-père et de la mère de Bernhard. Der Atem est un grand témoignage sur les liens d'amitié profonde qui unissaient le romancier à son aïeul. Dans ce récit, Bernhard décrit l'hospitalisation de son grandpère à Salzbourg, suivie de sa mort inopinée à la suite d'une erreur de diagnostic. Bernhard lui-même, âgé de dix-huit ans à cette époque, a été hospitalisé quelques jours après Freumbichler: «ce n'était rien qu'une pure conséquence logique que je fusse moi-même tombé malade après que mon grand-père fut soudain tombé malade » (S 11). À première vue, on a l'impression que l'adolescent se confond consciemment avec son 
grand-père pour rester proche de lui. Pourtant, ayant assisté à plusieurs décès dans son entourage, Bernhard décide d'emprunter le chemin de la guérison, ne serait ce que pour « contraindre l'infirmière [...] qui était certainement dans l'attente de ma mort » (S 22). Aussi déclare-t-il: «Je pense : soudain la respiration de l'homme devant moi s'est arrêtée. Je pense : je ne veux pas mourir » (S 21). Et plus tard, Bernhard ajoute : "le chemin de la vie a l'avantage d'être le résultat d'une autodétermination" (S 23). Mais pour parvenir à cette conclusion, il lui faut d'abord connaître l'atmosphère étouffante de l'hôpital de Salzbourg.

7 L'instinct de survie dont Bernhard fait preuve devient encore plus évident après le décès de son grand-père: "J'ai suivi mon grand-père dans la maladie, mais pas plus loin » (S 90) dit-il. Ainsi Bernhard s'aperçoit-il de l'importance qu'il y a à découvrir son indépendance personnelle. Il donne un témoignage éblouissant de cette décision, compréhensible il est vrai, uniquement à partir de ce deuil, qui lui fut indispensable à la réussite de son détachement par rapport à son grand-père :

Comme je l'avais vu tout à coup, il était non seulement possible d'être seul et de continuer sa route avec ses seules forces, mais c'était une stimulation incroyable de l'existence, inconnue auparavant. La mort de mon grand-père, si affreuse qu'elle fût apparue et si affreux qu'aient dû être tous les effets qu'elle avait exercés surmoi, cette mort avait été aussi une libération. Pour la première fois de ma vie j'étais libre et j'avais tiré pour moi-même une utilité de cette liberté totale soudainement ressentie en un sens qui, comme je le sens aujourd'hui, m'avait sauvé la vie. Dès l'instant où j'avais reconnu cela et où je l'avais mis en pratique, j'avais été gagnant dans les démêlés avec ma maladie. (S 91)

Après la disparition de Freumbichler, Bernhard, tout en manifestant son sens de la combativité, s'ouvre à la solitude qui lui sera nécessaire pour trouver sa propre voie artistique : «J'eus absolument le sentiment d'être sauvé à partir du moment où j'avais reconnu les possibilités de la parfaite solitude et où je me les étais appropriées. [...] Une seconde existence, une nouvelle vie et plus précisément une vie où j'étais complètement réduit à mes propres ressources, m'étaient ouvertes» (S 91). Dans les années suivantes, Bernhard développera en effet sa propre esthétique qui n'aura rien de commun avec l'univers antimoderniste et pieux, célébré par les romans de Freumbichler.

8 A l'instar de la perte de son grand-père qui fut un événement aussi douloureux que décisif dans la vie de l'adolescent, la mort prématurée de sa mère, racontée dans Die Kälte, représente elle aussi une tragédie bien que la relation entre mère et fils fût extrêmement compliquée. À l'inverse de Freumbichler qui éprouvait beaucoup d'affection pour son petit-fils, la mère de Bernhard était plutôt hostile envers son fils. A en croire les mots de ce dernier, il devait aller tout seul à la mairie pour retirer les cinq marks que l'Etat payait par mois pour son «entretien " (F 69). Dès sa toute première enfance, Bernhard se sent alors repoussé par une mère quine cesse de déverser un flot de malédictions sur lui. "Quand elle me voyait, elle voyait mon père, son amant, qui l'avait abandonnée. Elle ne voyait en moi que trop nettement le destructeur de sa vie [...] » (F 37). Pourtant, au fil des années, ils se sont rapprochés l'un de l'autre, pendant les périodes où Bernhard a été hospitalisé. C'est ainsi que le décès soudain de son grand-père et le cancer de sa mère ont contribué à cette réconciliation. La mort de la mère nous renvoie en quelque sorte à la disparition du grand-père. D'abord Bernhard se sent au bout de ses forces vitales. Suivant le cortège funèbre à l'occasion de l'enterrement de sa mère, il a non seulement l'impression d'avoir «tout perdu », mais aussi que sa «vie a perdu tout son sens » (F 122). Comme après la mort de son grand- 
père, Bernhard parvient finalement à vaincre sa profonde tristesse. Ce rebondissement de son élan vital est encore une fois lié à son combat contre sa propre maladie : « Tout à coup je ne voulus plus seulement améliorer mon état, je formulai l'exigence suprême : recouvrer ma santé » (F 124). Encore une fois, l'événement de la mort suscite la volonté de vivre chez le jeune Thomas Bernhard. Aussi prend-il l'initiative de chanter, activité à laquelle il tenait beaucoup: "je nourrissais un désir insatiable et inflexible de ces heures de musique à l'église. [...] La pratique de la musique était tout à coup mon entraînement vital » (F 131).

9 Face à la perte des êtres aimés et aux moribonds qui l'entourent dans divers hôpitaux et maisons de convalescence Bernhard ne s'abandonne jamais ni au désespoir ni à la maladie. Bien au contraire, l'omniprésence de la mort l'endurcit et l'amène à lutter contre les épreuves du sort. Si son acharnement à vivre est au cœur de son intransigeance inouïe, c'est surtout à travers son amour pour la musique et finalement sa vocation d'écrivain que Bernhard réussit à insuffler une vigueur nouvelle à sa vie.

Afin de mieux comprendre le choix du chemin de l'écriture qui s'est imposé à Bernhard, il reste à étudier la problématique de son père absent. Loin de chercher à élucider l'acte créateur uniquement à partir d'un événement tiré de sa biographie, on peut cependant dire qu'à côté de la thématique de la mort et de la maladie, la quête du père représente un aspect crucial dans les récits autobiographiques. Que Bernhard nous révèle ses véritables sentiments concernant son père où qu'il s'amuse à inventer des histoires, cela nous indiffère. Regardons plutôt la manière dont il essaie d'éclaircir l'énigme indéchiffrable que lui pose la disparition de son père. Nous voilà alors face à une quête interminable et un débat impossible à clore dont Bernhard tire sa créativité. Ainsi la problématique d'un questionnement insoluble devient-elle fondamentale chez lui, le moteur même de son écriture. Dans un entretien avec le journaliste Sepp Dreissinger, Bernhard explique: "Jusqu'à aujourd'hui, il n'y a pas eu de réponse définitive. Heureusement, car si les hommes n'avaient plus de questions à poser, il faudrait fixer le point final en dehors de l'univers ${ }^{4}$. » Et dans la même veine, dans Marcher, Bernhard écrit : «La vie toute entière n'est faite que de questions, parce que, constamment, nous n'existons que par les questions que nous posons, mais nous n'obtenons pas de réponse ${ }^{5}$.» Plus précisément, l'entreprise littéraire de l'Autrichien s'inscrit dans ces deux énoncés de Maurice Blanchot: «La question est le désir de la pensée » et « la réponse est le malheur de la question ${ }^{6}$.»

11 L'un des exemples les plus douloureux mettant à jour la vanité des efforts de Bernhard pour trouver une réponse, nous ramène à son premier récit Ein Kind: «Dès mes premières années j'avais renoncé à m'enquérir de mon père. Immédiatement tout le monde se fâchait contre moi [...]» (E 38). Si Bernhard est en mesure de s'expliquer l'aversion de sa mère contre l'homme qui l'avait rendu enceinte, puis abandonnée, il ne comprend pourtant pas pourquoi son grand-père, le "grand fournisseur d'explications » (E 72) se renferme dans le silence : " pourquoi mon grand-père aussi se taisait, c'était pour moi une énigme et ce l'est resté pour moi jusqu'à aujourd'hui » (E 39).

12 La quête menée par Bernhard pour démystifier ses origines paternelles est surtout abordée dans Die Kälte, son dernier récit autobiographique. Sa famille évitant le sujet de son père, Bernhard prit tout seul l'initiative de dévoiler le secret qui l'entoure. Il finit par trouver son grand-père paternel mais celui-ci dénonça son fils de la même façon que la mère de Bernhard : « celui-là s'est marié en Allemagne, il a fait cinq enfants et il 
s'est évaporé depuis longtemps » (F 71). Et pourtant, grâce au vieil homme, Bernhard se procura une photographie de son père, l'unique souvenir de la personne dont il ne devait jamais faire la connaissance. Mais à peine eut-il montré la photo à sa mère qu'elle la lui arracha des mains, puis la jeta dans le poêle. Après ce geste brutal, Bernhard décida de ne jamais évoquer la question de son père: "Jamais plus après cette explication, que j'ai en mémoire comme l'une des pires de ma vie, je n'avais fait mention de mon père à la maison » (F 71).

Telle a été son existence, réduite à une vie de questions qui se brisent contre un mur de silence. Chaque fois que Bernhard s'approche de la question maudite, il se trouve devant une porte fermée. C'est aussi le cas lorsqu'il cherche à rejoindre une ancienne amie d'école de ses parents ${ }^{7}$. Pourtant, la rencontre de ces deux personnes n'aura jamais lieu : un jour avant leur rendez-vous Bernhard lit dans un journal qu'elle a été tuée dans un accident de voiture :

Mais un jour avant la rencontre je découvrais dans le journal une image épouvantable: deux cadavres décapités sur une route d'accès à Salzbourg; la camarade d'école de ma mère, la seule qui eût pu me renseigner sur mon père avait été victime d'un accident mortel. Cette image d'épouvante dans le journal m'avait donné une certitude : je ne devais plus m'informer sur mon père. (F 68-69) ${ }^{8}$

\section{Dans son excellent livre, Louis Huguet n'a pas manqué de commenter :}

Chaque fois cependant que l'écrivain sera sur le point de percer le mystère, la mort surgira: celle de témoins de toute première importance, finalement la sienne propre. Mais a-t-il vraiment souhaité «savoir»? Le Sphinx qui pose l'énigme insoluble et mortelle n'est-il pas au tréfonds de nous-même à la fois pour stimuler la quête et pour nous mettre en garde contre la violation du secret? Curiosité et angoisse. Finalement intervient la brutalité du destin ${ }^{9}$.

Si Bernhard n'a jamais réussi à rencontrer la femme qui aurait pu l'aider à percer l'aura mystérieuse de son père, nous pouvons ajouter une autre "énigme insoluble ", mentionnée par Huguet dans son étude. Car au cours de ses recherches inépuisables sur la vie de l'auteur, Huguet a même fini par retrouver la demi-sœur de Bernhard, née du deuxième mariage du père. Huguet arriva finalement à la contacter. Bernhard, lui, ne l'avait jamais évoquée. Lorsque Huguet tenta de lui faire part de cette nouvelle, c'était trop tard: Bernhard venait lui aussi de mourir. Pourtant, Peter Fabjan, demi-frère de Bernhard du côté maternel, fit remarquer que ce dernier était peut-être au courant de l'existence de sa demi-sœur ${ }^{10}$. Au demeurant, Huguet n'hésite pas non plus à nous révéler les circonstances de la conception de Bernhard; selon lui, la mère de l'écrivain aurait probablement été violée par son ami Alois Zuckerstätter.

Si ces révélations ont certainement contribué à nous sensibiliser davantage à l'obscur milieu familial dont Bernhard est issu, le silence ultime du Sphinx ne sera jamais brisé. Le passé de Bernhard semble toujours destiné à conserver son aspect obscur. Dans cette perspective, le silence du Sphinx est encore plus ambivalent : au-delà de Bernhard ne descendant jamais, de son vivant, dans les profondeurs inouïes du gouffre familial, ou du chercheur incapable de nous fournir la quiétude d'une certitude confortable, ce qui compte c'est l'absence d'explication définitive à toute histoire fictive et biographique. Et rappelons que la prose de Bernhard est toujours marquée par cette confusion entre fiction et autobiographie. En attendant l'hypothétique divulgation du dernier secret du Sphinx, nous ne connaîtrons jamais la vérité sur la vie de Bernhard, tant il est vrai que nous respectons l'absence d'éclaircissement final. 
17 Et nous voilà constamment ramenés aux mêmes questions: Bernhard a-t-il véritablement cherché à connaître l'histoire de son père ? Ou bien, ne se contente-t-il pas, plutôt, de nous dissimuler ses intentions personnelles? Le labyrinthe des questions demeure sans issue aucune. C'est précisément cette énigme qui inspire autant son écriture que notre lecture de ses œuvres. L'appréciation éblouissante que donne Maurice Blanchot de la question convient parfaitement dans ce contexte :

La question attend la réponse, mais la réponse n'apaise pas la question et, même si elle y met fin, elle ne met pas fin à l'attente qui est la question de la question. Question, réponse, nous trouvons entre ces deux termes l'affrontement d'un rapport étrange, dans cette mesure où la question appelle, en la réponse, ce qui lui est étranger et en même temps veut se maintenir dans la réponse comme ce tour de la question que la réponse arrête pour mettre fin au mouvement et donner le repos. Seulement la réponse, répondant, doit reprendre en elle l'essence de la question, qui n'est pas éteinte par ce qui y répond ${ }^{11}$.

\section{NOTES}

1. Thomas Bernhard, Le Froid, Une mise en quarantaine, tr. Albert Kohn, Paris, Gallimard, 1984, la lettre $\mathrm{F}$ renvoie systématiquement à cette édition.

2. Ibid., Le Souffle, Une décision, tr. Albert Kohn, Paris, Gallimard, 1983, la lettre S renvoie systématiquement à cette édition.

3. Ibid., Un Enfant, tr. Albert Kohn, Paris, Gallimard, 1984, la lettre E renvoie systématiquement à cette édition.

4. Sepp Dreissinger, Von einer Katastrophe in die andere. 13 Gespräche mit Thomas Bernhard, Wien, Bibliothek der Provinz, 1992, p. 113.

5. Thomas Bernhard, Amras et autres récits, tr. J.-C. Hémery et E. Kaufholz, Paris, Gallimard, 1987, p. 217.

6. Maurice Blanchot, L'Entretien infini, Paris, Gallimard, 1969, p. 14-15.

7. Louis Huguet offre de plus amples détails sur ces circonstances. Voir Louis Huguet, Thomas Bernhard ou le silence du sphinx: recherches biographiques bernhardiennes, Perpignan, Presses universitaires de Perpignan, 1991.

8. Urs Bugmann parle de cet incident en citant Le Froid: «les question subsistaient, elles se multipliaient avec le temps, avec la maladresse de mon existence, avec ma volonté de connaissance, [...] Je n'avais jamais cessé d'arriver aux preuves, toute ma vie j'avais été en quête de preuves pour mon existence, avec une intensité un jour plus grande, un jour moins grande mais toujours avec insistance et avec conséquence, cependant si j'avais des preuves de ce genre dans les mains [...] elles n'étaient pourtant pas suffisamment solides [...] elles se révélaient un recul» (F 75-76). Bugman choisit un passage qui nous renvoie également à la lutte menée par les personnages de Bernhard s'efforçant de comprendre le monde extérieur. Pourtant, la conclusion de Bugmann semble plutôt simpliste. En commentant l'épisode cité ci-dessus, il constate: «L'homme livré à lui-même est incapable de trouver un point d'ancrage ni un cadre, car on ne peut véritablement déterminer ses origines qui demeurent insondables». Urs Bugmann, Bewältigungsversuch: Thomas Bernhards autobiographische Schriften, Frankfurt, Peter Lang, 1981, p. 342. Le mot «recul/Rückschritt» peut être interprété dans le contexte de la futilité chez 
Bernhard qui attend une réponse définitive. Bernhard semble être plus dialectique que Bugmann. Il comprend finalement à quel point il est vain de croire à l'existence d'une explication finale.

9. Huguet, op. cit., p. 66.

10. Ibid., p. 170 , note en bas de page \#105.

11. Maurice Blanchot, L'Entretien infini, Paris, Gallimard, 1969, p. 16.

\section{RÉSUMÉS}

Déconcertés par les thèmes morbides de Thomas Bernhard, beaucoup de critiques les ont analysés comme étant le produit de la résignation des personnages face à eux-mêmes et face au monde extérieur. Contrairement à ces critiques, nous essayerons de mettre en valeur la rigueur du Moi dans les récits autobiographiques L'Enfant, Le Souffle et Le Froid. Dans ces œuvres, il s'agit d'un Moi - celui du jeune Thomas Bernhard - qui se livre à un combat acharné contre les épreuves du destin. Face à la perte des êtres aimés ou à sa maladie, Bernhard ne s'abandonne jamais au désespoir. À travers son amour pour la musique et finalement sa vocation d'écrivain, il réussit à insuffler une vigueur nouvelle à sa vie. Cette vigueur devient surtout sensible dans la quête menée par Bernhard pour trouver son père. Si cette quête débouche sur un labyrinthe de questions insolubles, elle inspire surtout la perpétuation de l'écriture.

Bei der Betrachtung der Sekundärliteratur über Thomas Bernhards Werk stößt man immer wieder auf Kritiker, die - durch die oftmals morbiden Themen in äußerste Verwirrung gebracht dem Autor vorwerfen, daß er lediglich Figuren beschreibe, denen der Weg aus der Resignation versagt bleibe. Um diesem weit verbreiteten Vorwurf entgegenzuwirken, werde ich die Unerbittlichkeit und Unerschrockenheit des Ichs in den autobiographischen Erzählungen Ein Kind, Der Atem und Die Kälte hervorheben. In diesen drei Werken stößt der Leser auf ein Ich - den jungen Thomas Bernhard - welches unermüdlich gegen die ihm vom Schicksal auferlegten Prüfungen ankämpft. Ganz gleich ob es sich um den schmerzlichen Verlust ihm nahestehender Personen oder aber um die an ihm zehrende Tuberkulose handelt, Bernhard gibt sich niemals vollkommen der Verzweiflung anheim. Dank seiner Liebe zur Musik und schließlich seiner Entscheidung Schriftsteller zu werden, gelingt es ihm neue Kraft für sein Leben zuschöpfen. Diese Lebenskraft äußert sich insbesondere in Bernhards Suche nach seinem Vater. Auch wenn diese Suche Bernhard immer wieder vor unlösbare Rätsel führt, so ist die Frage nach dem Vater letztendlich auch die Triebfeder für den Schreibprozeß. 